7th International Workshop on Astronomy and

Relativistic Astrophysics (IWARA 2016)

International Journal of Modern Physics: Conference Series

Vol. 45 (2017) 1760019 (5 pages)

(C) The Author(s)

DOI: $10.1142 / S 2010194517600199$

\title{
Quadrupole Mass Optimization in Very High Speed Using FEM: First Attempt
}

\author{
Paulo R. M. Nogueira, Carlos Frajuca, Daniel Coppedé, Fabio S. Bortoli, \\ Givanildo A. Santos, and Francisco Y. Nakamoto \\ Departmento de Mecânica \\ Instituto Federal de Educação, Ciência e Tecnologia de São Paulo (IFSP) \\ Rua Pedro Vicente, 625, 01109-010 São Paulo, SP, Brazil \\ frajuca@ifsp.edu.br,danielcoppede@gmail.com,bortoli.fabio@gmail.com, \\ givanildo@ifsp.edu.br,nakamoto@ifsp.edu.br
}

Published 15 August 2017

\begin{abstract}
A experiment to measure the velocity of gravity is been planned. In order to achieve a reasonable signal in the detector, a very massive quadrupole mass is put to rotate at a very high rotation speed. In order to maximize the signal, the mass much be as higher as possible. This work test mass geometries in Finite Element Modeling (FEM) with the goal to maximize the resistance to high rotation speed.
\end{abstract}

Keywords: Gravity Speed, Ultrahigh Speed, Control, Vacuum, Magnetic Bearing.

PACS numbers: 04.30.Db and 04.80.Cc

\section{Introduction}

The speed of gravity is one of the great mysteries. A way to detect it would be through the detection of gravitational waves but this is very difficult because until today only one were detected, but besides the detection of such wave a light counterpart must be found at the same time with different instruments, which makes this option very challenging. It is best to issue a signal and detect it, which is intended in the continuation of this project.

In order to achieve this, it is proposed to develop a rotating system running at 600,000 rotations per minute (rpm), this presents great challenge in the parts of magnetic suspension, direct drive, control of rotating systems and highly resistant materials.

This is an Open Access article published by World Scientific Publishing Company. It is distributed under the terms of the Creative Commons Attribution 4.0 (CC-BY) License. Further distribution of this work is permitted, provided the original work is properly cited. 


\section{Theoretical Foundation}

The velocity of gravitational waves in the theory of general relativity equals the velocity of light in vacuum, $c$ (see Ref. ${ }^{1}$ ) Formally, $c$ is a conversion factor for changing the unit of time to the unit of space. ${ }^{2}$ Newtonian gravitation is described by the Poisson equation, whereby, when the mass distribution of a molting system, its gravitational field fits instantaneously. Therefore, the theory assumes that the speed of gravity is infinite. Until the 19th cenury only an anomaly in astronomical observations could not be reconciled with the Newtonian gravitational model of instantaneous action: the French astronomer Urbain Le Verrier determined in 1859 that the elliptical orbit of the planet Mercury precessed at a rate significantly different from that predicted by the Newtonian theory. ${ }^{3}$

The first attempt to combine a finite gravitational velocity with Newton's theory was made by Laplace in 1805. Based on Newton's law of force he considered a model in which the gravitational field is defined as a field of radiation or a fluid. Changes in the movement of the capture body are transmitted by some type of waves. ${ }^{4}$

In 1900 Hendrik Lorentz attempted to explain gravity based on his ether theory and Maxwell's equations. After proposing (and rejecting) a Le Sage type model, he assumed as Ottaviano Fabrizio Mossotti and Johann Karl Friedrich Zöllner that the atraction of opposite charged particles is stronger than the repulsion of equal charged particles. The resulting net force is exactly what is known as universal gravity, in which the speed of gravity is that of light. This leads to a conflict with the law of gravity of Isaac Newton in which it was demonstrated by Pierre Simon Laplace that a finite velocity of gravity leads to some kind of aberration and therefore causes the orbits to be unstable. However, Lorentz showed that the theory is not covered by Laplace's critique because of the structure of Maxwell's equations only effects in the order $v^{2} / c^{2}$ arise. ${ }^{5}$

General relativity predicts that gravitational radiation should exist and propagate like a wave at the speed of light: a gravitational field evolving slowly and weakly would, according to general relativity, produce effects like those of Newtonian gravitation.

The first direct observation of gravitational waves, from the fusion of a pair of black holes, on September 14, 2015 (announced by the LIGO and Virgo collaborations on February 11, 2016 (see Refs. ${ }^{6-8}$ )) allowed a more direct measurement of its speed. Other attempts are made to detect gravitational waves ${ }^{9-15}$.

\section{This Experiment}

Therefore, to directly measure the velocity of gravity, a tidal gravitational signal emitter and a detector are proposed to receive this signal. The speed will be obtained by the phase difference between the two devices weighted by distance. Since the speed of this signal must be very high, the device must rotate at a very high speed. The calculations to obtain the signal strength at the detector are shown below.

In Fig. 1 the system is shown. 


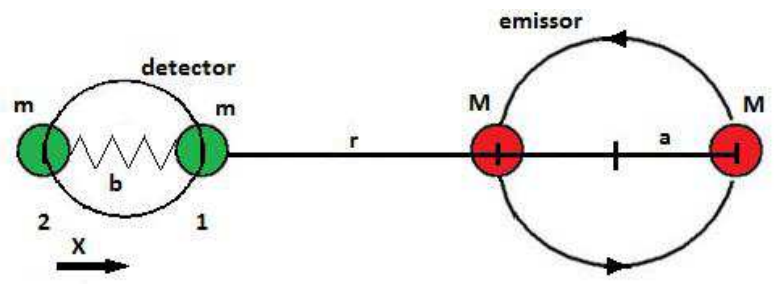

Fig. 1. The signal generator is a quadruple mass spinning at very high speeds, and the signal phase is measured at the detector and the velocity is obtained using the phase difference and the distance $r$.

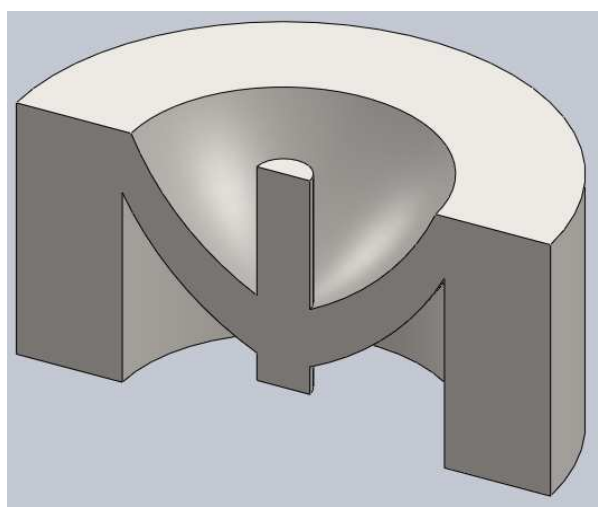

Fig. 2. Analyzed model.
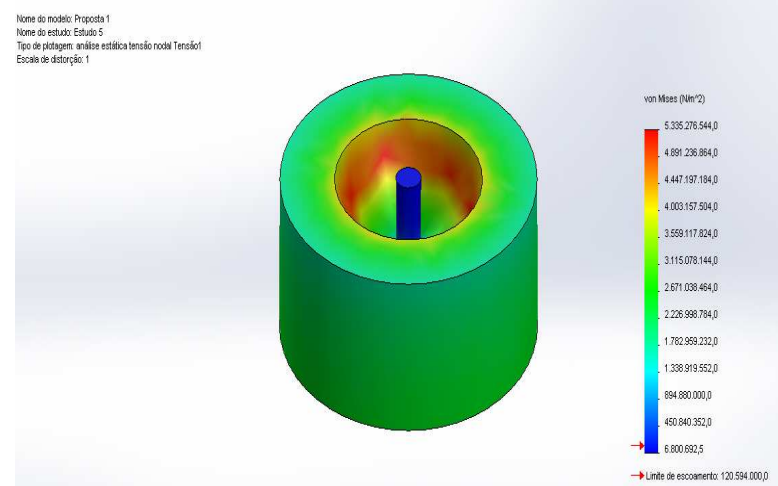

Fig. 3. Von Mises stress results. 
P. R. M. Nogueira et al.

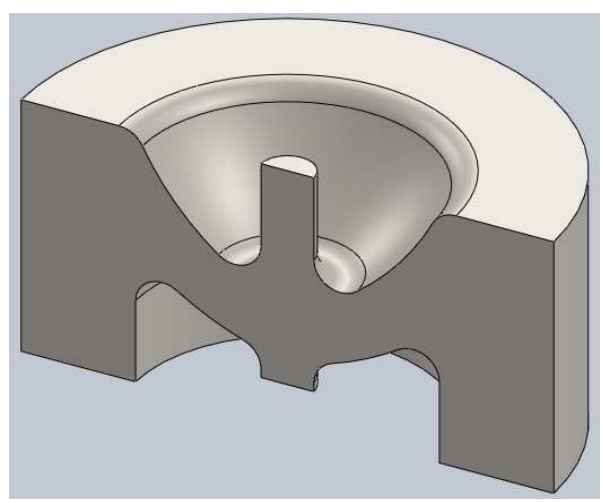

Fig. 4. New proposed model.

\section{Methodology}

The idea is to make a quadruple mass made of carbon fiber and other materials. For the start a more simple model is tested in FEM. As the FEM code does not support carbon fiber we choose graphite. Which have the same density then it is necessary to compare the maximum Von Mises Stress. Fig. 2 shows the analysed model, and Fig. 3 shows the Von Mises Stresses obtained in the simulation.

\section{Conclusion}

After the simulations we concluded that the sharp edges cause tension building. Then, it is necessary to avoid sharp edges in the model. Fig. 4 shows the new proposed model with no sharp edges. The next step is to simulate a composed model with a quadruple mass component.

\section{Acknowledgments}

\section{References}

1. J. B. Hartle. Gravity: An Introduction to Einstein's General Relativity. (AddisonWesley, Boston, 2003) ISBN 981-02-2749-3, p. 332.

2. U. Le. Verrier Lettre de M. Le Verrier à M. Faye Sur la Théorie de Mercure et Sur le Mouvement du Périhélie de Cette Planéte C. R. Acad. Sci. 49, 379 (1859).

3. P. S. Laplace. A Treatise in Celestial Mechanics, Vol. IV, Book X, Chapter VII (1805), translated by N. Bowditch (Chelsea, New York, 1966).

4. J. Zenneck. Gravitation, Encyklopädie der Mathematischen Wissenschaften mit Einschlussihrer Anwendungen (in German), (doi: 10.1007/978 - 3-663-16016 - 8_2) Vol. 5, 25 (1903).

5. H. A. Lorentz. Considerations on Gravitation, Proc. Acad. Amsterdam 2, 559 (1900).

6. LIGO Scientific Collab. and Virgo Collab. (B. P. Abbott et al.), Phys. Rev. Lett., 116 (6), 061102 (2016). Observation of Gravitational Waves from a Binary Black Hole Merger. arXiv:1602.03837(2016) (PDF). 
7. D. Castelvecchi and A. Witze. Einstein's Gravitational Waves Found at Last. Nature News doi:10.1038/nature.2016.19361. Retrieved 11 February 2016.

8. The Editorial Board. The Chirp Heard Across the Universe. New York Times. Retrieved 16 February 2016.

9. C. Frajuca, K. L. Ribeiro, O. D. Aguiar, L. A. Andrade, P. J. Castro, N. S. Magalhaes, and R. M. Marinho Jr. CQG 21, (2004).

10. C. Frajuca, K. L. Ribeiro, L. A. Andrade, W. F. Velloso Jr., J. L. Melo, O. D. Aguiar, and N. S. Magalhaes, CQG 19, 1961 (2002).

11. W. W. Johnson and S. M. Merkowitz, Phys. Rev. Lett. 70, 2367 (1993).

12. W. W. Johnson and S. M. Merkowitz, Phys. Rev. D 56, 7513 (1997).

13. S. M. Magalhaes, O. D. Aguiar, W. W. Johnson, and C. Frajuca, Gen. Relat. Grav. 29, 1511 (1997).

14. N. S. Magalhaes, W. W. Johnson, C. Frajuca, and O. D. Aguiar, MNRAS 274, 670 (1995).

15. N. S. Magalhaes, W. W. Jonhson, C. Frajuca, and O. D. Aguiar, Astrophysical Journal 475, 462 (1997). 